\title{
MMP-2 and MMP-9 and Their Tissue Inhibitors in the Plasma of Preterm and Term Neonates
}

\author{
CHRISTINA G. SCHULZ, GRZEGORZ SAWICKI, ROBERT P. LEMKE, BIRGITTE M. ROETEN, \\ RICHARD SCHULZ, AND PO-YIN CHEUNG \\ Department of Pharmacology [C.G.S., G.S., R.S., P.-Y.C.], Department of Pediatrics [R.P.L., R.S., \\ P.-Y.C.], Perinatal Research Centre [B.M.R., R.S., P.-Y.C.], University of Alberta, Edmonton, Alberta, \\ Canada T6G $2 R 7$
}

\begin{abstract}
ABSTR
Matrix metalloproteinases (MMP) and their tissue inhibitors
(TIMP) are involved in a variety of physiologic growth and
development and pathophysiologic inflammatory conditions. We
hypothesized that 1) MMP-2 and -9 plasma activities and
TIMP-1 and -2 plasma concentrations in preterm and term
neonates were dependent on the gestational and postnatal age;
and 2 ) the respective MMP and their inhibitors were deranged in
the development of bronchopulmonary dysplasia (BPD) and
intraventricular hemorrhage (IVH) in preterm neonates. From
1998 to 1999 , blood samples were collected from preterm neo-
nates (25-36 wk gestation) with or without BPD and/or IVH as
well as from healthy term (37-40 wk gestation) neonates during
the first 28 d of life. MMP-2 and MMP-9 plasma activities were
measured by zymography; TIMP-1 and TIMP-2 plasma concen-
trations were determined by ELISA. In neonates without BPD or
IVH ( $n=50$ ), MMP-2 and MMP-9 plasma activities both
appeared to be gestational age dependent, with the highest levels
observed in neonates of 33-36 wk gestation. TIMP-1 plasma
concentration was highest in term neonates but no gestational
\end{abstract}
difference was found in TIMP-2. Only MMP-9 showed a 50\% decrease after $\mathrm{d} 1$ in the first postnatal month. Twelve preterm infants with BPD and/or IVH had significantly lower MMP-2 but higher MMP-9 activity and higher TIMP-1 concentration than those of corresponding neonates without BPD or IVH. These findings show the gestational age-dependent expression of plasma MMP activities and their inhibitors. MMP and TIMP may be involved in the feto-neonatal development and may contribute to the pathogenesis of BPD and/or IVH in critically ill preterm neonates. (Pediatr Res 55: 794-801, 2004)

Abbreviations
MMP-2, matrix metalloproteinase-2 (EC 3.4.24.24)
MMP-9, matrix metalloproteinase-9 (EC 3.4.24.35)
TIMP-1, tissue inhibitor of matrix metalloproteinases-1
TIMP-2, tissue inhibitor of matrix metalloproteinases-2
BPD, bronchopulmonary dysplasia
ECM, extracellular matrix
IVH, intraventricular hemorrhage

MMP are a group of zinc-dependent endopeptidases involved in the process of tissue remodeling through the degradation of the ECM. Tissue remodeling occurs in various physiologic conditions, such as embryogenesis and wound healing, as well as in pathologic conditions, including inflammatory diseases, tumor cell invasion, and angiogenesis (1). Among the MMP, MMP-2 (gelatinase A: pro-enzyme, $72 \mathrm{kD}$, constitutive) and MMP-9 (gelatinase B: pro-enzyme, $92 \mathrm{kD}$, inducible) are of particular interest because of their novel roles other than tissue remodeling. Recently, MMP-2 has been found to play a role in acute disease processes such as platelet aggregation (2),

Received February 10, 2003; accepted December 8, 2003

Correspondence: Po-Yin Cheung, MBBS, Ph.D., FRCPCH, FRCP, NICU Royal Alexandra Hospital,10240 Kingsway Avenue, Edmonton, AB, Canada T5H3V9; e-mail: poyin@ualberta.ca

Supported by a grant-in-aid from the Stollery Children's Hospital Foundation.

P.-Y.C. and R.S. are clinical investigator and senior scholar of the Alberta Heritage Foundation for Medical Research, respectively.

DOI: 10.1203/01.PDR.0000120683.68630.FB the regulation of vascular tone (3), and myocardial dysfunction after ischemia-reperfusion (4). Whereas both MMP-2 and MMP-9 are activated through proteolytic cleavage and/or oxidative stress (5), the proteolytic function of MMP is at least in part inhibited by tissue inhibitors of MMP (TIMP) (6). Thus, imbalances between MMP and TIMP may result in an increased proteolytic activity and therefore promote the progression of pathologic conditions such as inflammatory and myocardial diseases $(1,4)$ and intracerebral hemorrhage (7).

Both term and preterm neonates have to undergo various adaptive processes to environmental conditions ex utero, including an enhanced oxidative stress. However, the organ systems of preterm neonates may have not sufficiently developed to encounter conditions ex utero. There is no information regarding the developmental and postnatal changes of MMP and TIMP during the feto-neonatal period in humans. We therefore investigated normative values for MMP-2 and MMP-9 and their inhibitors-TIMP-1 and TIMP-2 in preterm and term neonates and the respective relationship with gesta- 
tional age and in the early postnatal period. Preterm neonates have a diminished antioxidant capacity (8) and the postnatal exposure to high concentrations of reactive oxygen species in critically ill neonates may promote the development of "oxygen radical disease in neonatology" (9), which includes BPD and IVH. We speculated that MMP might play a role in the pathophysiological process of BPD and/or IVH via their actions on the remodeling of the ECM, vasomotor regulation, and platelet aggregation. Therefore, in this study we also investigated MMP-2 and MMP-9 and TIMP-1 and TIMP-2 in the development of BPD and IVH in preterm neonates. We hypothesized that 1) MMP-2 and -9 plasma activities and TIMP-1 and -2 plasma concentrations in preterm and term neonates were dependent on the gestational and postnatal age; and 2) the respective MMP and their inhibitors were deranged in the development of BPD and IVH in preterm neonates.

\section{MATERIALS AND METHODS}

The study received approval from the Health Research Ethics Board, University of Alberta.

Patient enrollment. Between October 1998 and August 1999, after informed parental consent, patients admitted to the neonatal intensive care unit at the Royal Alexandra Hospital, Edmonton, Canada, were enrolled into the study.

The patients were divided into four groups according to their gestational age as assigned by the obstetric assessment: group I (25-28 wk gestation), group II (29-32 wk gestation), group III (33-36 wk gestation), and group IV (37-40 wk gestation). The majority of patients were admitted for observation (group IV), prematurity, and hyaline membrane disease (groups I, II, III) and required short hospital stay. Preterm neonates ( $\leq 32 \mathrm{wk}$ gestation) who subsequently developed BPD and IVH were analyzed separately. BPD was diagnosed as the requirement of oxygen therapy at $28 \mathrm{~d}$ of postnatal age with radiologic evidence of chronic lung disease (10). Oxygen therapy was given and adjusted to achieve oxygen saturation between $88 \%$ and $92 \%$ by pulse oximetry (Nellcor Inc., Hayward, CA, U.S.A.). IVH was diagnosed using cranial ultrasonography, which was performed at least once in the first $2 \mathrm{wk}$ of life, and classified according to Papile's classification (11). These patients were then matched with patients within 2 wk limit difference in gestational age admitted during the study period, and diagnosed with the aforementioned conditions but not BPD or IVH.

Patients with sepsis (documented positive blood cultures), cardiovascular compromise (hypotension with mean arterial pressure $>2$ SD below normative mean for gestational age), and multiple organ failure were excluded. Demographic characteristics, including birth weight, gestational age, gender, cesarean section, use of antenatal steroids, Apgar score at 1 and 5 min of life, were recorded as well as the lowest platelet count, the duration of hospital stay, and oxygen therapy.

Sample collection. Whole venous or arterial blood samples $(100 \mu \mathrm{L})$ were collected in $3.15 \%$ tri-sodium citrate solution (9:1, v:v) on $\mathrm{d} 1,3,7,14$, and 28 of hospitalization in conjunction with routine blood work. The blood samples were immediately centrifuged $\left(1700 \times \mathrm{g}, 10 \mathrm{~min}\right.$ at $\left.4^{\circ} \mathrm{C}\right)$ and the plasma was removed and stored at $-80^{\circ} \mathrm{C}$ until further processing.

Measurement of MMP-2 and MMP-9 plasma activities. Gelatinolytic activities of plasma MMP were examined by zymography. The samples were mixed with SDS sample buffer and subjected to $8 \%$ polyacrylamide separating gels containing gelatin $(2 \mathrm{mg} / \mathrm{mL}$, Sigma Chemical, St. Louis, MO, U.S.A.). The supernatant of human fibrosarcoma HT 1080 cells (American Type Culture Collection, Manassas, VA, U.S.A.) was used as a standard for MMP. After electrophoresis, the gels were washed with $2.5 \%$ Triton X-100 and incubated overnight at $37^{\circ} \mathrm{C}$ in $50 \mathrm{mM}$ Tris- $\mathrm{HCl}$ buffer $\left(0.15 \mathrm{M} \mathrm{NaCl}, 5 \mathrm{mM} \mathrm{CaCl}_{2}\right.$, $50 \mathrm{mM}$ Tris $\mathrm{HCl}$, and $0.05 \% \mathrm{NaN}_{3}, \mathrm{pH}$ 7.6). Gelatinolytic activities were visualized as transparent bands against a blue background through staining with Coomassie Blue (72 and 92 $\mathrm{kD}$ for MMP-2 and -9 , respectively, and $64 \mathrm{kD}$ for the active proteolyzed form of MMP-2). In this study, we did not identify any gelatinolytic activity at $64 \mathrm{kD}$. Photographs of the zymograms were scanned using a ScanJet scanner (Hewlett Packard, Palo Alto, CA, U.S.A.). The intensity of the digitalized bands was analyzed by SigmaGel measurement software (Jandel Scientific, San Rafael, CA, U.S.A.) and MMP activity was calculated per amount of protein. The plasma protein concentration was assessed by bicinchoninic acid assay (Sigma Chemical) using BSA as a standard.

Determination of TIMP-1 and TIMP-2 plasma concentrations. TIMP-1 and TIMP-2 plasma concentrations were determined by commercially available enzyme immunoassays (Oncogene Research Products, affiliated with Merck, Darmstadt, Germany). The samples were pipetted into wells containing a specific antibody to TIMP-1 or TIMP-2, respectively. Unbound material was then washed away, followed by addition of a horseradish peroxidase conjugated to either TIMP-1 or TIMP-2 antibodies. After incubation and another washing step, a chromogenic substance (tetramethylbenzidine) was added to the wells, which was converted through horseradish peroxidase from a colorless to a blue solution turning yellow upon addition of stopping reagent. The intensity of color is proportional to the amount of TIMP-1 or TIMP-2 protein in the sample. Quantification of the reaction product was achieved by measuring the absorbance at 450 and $595 \mathrm{~nm}$ using a spectrophotometer. The concentration of TIMP-1 or TIMP-2 was determined by interpolation from a standard curve.

Statistics. Data are expressed as mean \pm SD. The results between different gestation groups were analyzed using either one-way ANOVA or one-way ANOVA on ranks, for parametric and nonparametric variables, respectively. Fisher's LSD and Dunn's tests were then used for posthoc testing when required. To analyze the differences between those neonates with BPD and IVH (Group A) and their gestation-matched controls (Group B), $t$ or rank sum tests were used. Differences between proportions were analyzed by $z$ test or ANOVA as appropriate. Results were considered significant at $p<0.05$.

\section{RESULTS}

During the study period, 65 patients were enrolled. Among these, three patients were excluded because of blood culture 
positive sepsis. Incomplete samples were collected for all time points after d 3 of life due to early discharge of healthy neonates. Among the enrolled patients, 50 did not develop BPD or IVH, and their clinical characteristics are shown in Table 1.

During the study period, a total of 12 preterm patients (19\%) were enrolled and subsequently developed BPD and/or IVH during hospitalization. Among these, four patients had BPD and four other neonates had IVH alone. Four patients in this group were diagnosed with both conditions. Three patients had grade 3 or $4 \mathrm{IVH}$, five had grade 2 or $1 \mathrm{IVH}$. Their characteristics are shown in Table 2.

Developmental changes in neonates (25-40 wk gestation) without BPD or IVH. The MMP-2 plasma activity in neonates without BPD or IVH appeared to peak at 33-36 wk gestation. Patients of 33-36 wk gestation had the highest MMP-2 plasma activity from $\mathrm{d} 1$ (Fig. $1 A, 1 B$ ) through $\mathrm{d} 7$, whereas MMP-2 plasma activity of patients of 37-40 wk gestation was significantly lower than that of preterm neonates (29-32 and 33-36 wk gestation) (d 3:16 $\pm 4,20 \pm 7,22 \pm 8$, and $7 \pm 5$ arbitrary units/mg protein of groups I, II, III, and IV, respectively, $p<$ $0.05 ; \mathrm{d} 7: 19 \pm 6,15 \pm 5$, and $24 \pm 10$ arbitrary units $/ \mathrm{mg}$ protein of groups I, II, and III, respectively, $p=0.05$ ). No significant differences were found between gestational age groups in the plasma samples of d 14 and $\mathrm{d} 28$ in these neonates. We also observed that MMP-9 plasma activity followed a pattern similar to MMP-2, revealing the highest levels of MMP-9 activity in neonates who were born at 33-36 wk gestation. The MMP-9 plasma activity was significantly higher in these neonates than in any other groups on $\mathrm{d} 1$ (Fig. 1C) and d 3 (data not shown). Similar trends persisted on d 7 and d 14, but the differences were not significant (d 7: $46 \pm 55,23 \pm 19$, and $2 \pm 1$ arbitrary units/mg protein of groups III, II, and I, respectively; d 14: $40 \pm 25,21 \pm 36$, and $4 \pm 1$ arbitrary units $/ \mathrm{mg}$ protein of groups III, II, and I, respectively; $p=0.07$, $\beta=0.79$ ).

The TIMP-1 plasma concentrations in neonates without BPD or IVH were significantly elevated on d 1 only in
Table 2. Clinical characteristics of preterm neonates who developed BPD and/or IVH (Group A), and their gestational age-matched controls who did not develop BPD or IVH (Group B)

\begin{tabular}{lcc}
\hline & Group A & Group B \\
\hline No. & 12 & 12 \\
Gestational age (wk) & $27.3 \pm 1.6$ & $29.0 \pm 1.6$ \\
Birth weight (g) & $942 \pm 332$ & $1156 \pm 288$ \\
Male gender (\%) & 50 & 67 \\
Delivered by cesarean section (\%) & 33 & 42 \\
Use of antenatal steroids (\%) & 100 & 89 \\
Apgar score at 1 min & $6.5(4-7.5)$ & $6(6-7.5)$ \\
Apgar score at 5 min & $8(7-9)$ & $8(7.5-9)$ \\
Primary diagnosis & & \\
$\quad$ Prematurity & 2 & 4 \\
HMD & 7 & 5 \\
IUGR & - & 2 \\
Twin & $190 \pm 112$ & $187 \pm 71$ \\
Lowest platelet count $\left(\times 10^{9} / \mathrm{L}\right)$ & $511 \pm 563$ & $143 \pm 148$ \\
Duration of oxygen therapy (d) & $51 \pm 26$ & $35 \pm 21$ \\
Hospital stay (d) &
\end{tabular}

Values are given as means $\pm \mathrm{SD}$, percentages, or median (interquartile range).

HMD, hyaline membrane disease; IUGR, intrauterine growth retardation.

neonates of 37-40 wk gestation (Fig. 2A) compared with those of neonates of 29-32 wk gestation. On the other hand, we did not find any significant differences between gestational age groups in TIMP-2 plasma concentration on d 1 (Fig. $2 B$ ) or on any following day during the observation period.

Postnatal changes in preterm neonates (25-36 wk gestation) without BPD or IVH. Due to the short hospital stay of term neonates, we were unable to study the postnatal changes of MMP and TIMP in neonates of 37-40 wk gestation (group IV). MMP-2 plasma activity in preterm neonates (group I-group III) did not show postnatal changes during the observation period of the first $28 \mathrm{~d}$ of life (Fig. 3A, 3B). A 50\% decrease of MMP-9 plasma activity in these patients was observed after d 1 (Fig. 3C). MMP-9 plasma activity on $\mathrm{d} 1$ was more than 2 -fold compared with that of subsequent postnatal age, but the differences were not statistically significant $(p=0.08, \beta=0.67)$. We did not find any

Table 1. Clinical characteristics of 50 neonates who did not develop bronchopulmonary dysplasia or intraventricular hemorrhage

\begin{tabular}{|c|c|c|c|c|}
\hline & Group I & Group II & Group III & Group IV \\
\hline No. & 4 & 22 & 17 & 7 \\
\hline Gestational age (wk) & $26.7 \pm 1.2$ & $31.0 \pm 1.1$ & $34.1 \pm 1.1$ & $38.1 \pm 1.3$ \\
\hline Birth weight $(\mathrm{g})$ & $1127 \pm 201$ & $1495 \pm 384$ & $2138 \pm 358$ & $3238 \pm 511$ \\
\hline Delivered by cesarean section (\%) & 9 & 63 & 47 & 29 \\
\hline Use of antenatal steroids (\%) & 91 & 63 & 47 & 0 \\
\hline Apgar score at $1 \mathrm{~min}$ & $7.5(5-9)$ & $7(6-8)$ & $7(5-8)$ & $5(4-6.8)$ \\
\hline Prematurity & 4 & 5 & 9 & - \\
\hline HMD & - & 11 & 1 & 1 \\
\hline IUGR & - & 5 & 2 & - \\
\hline Twin & - & 1 & 5 & - \\
\hline Others & - & - & - & 6 \\
\hline Lowest platelet count $\left(\times 10^{9} / \mathrm{L}\right)$ & $229 \pm 24$ & $186 \pm 56$ & $218 \pm 55$ & $211 \pm 28$ \\
\hline
\end{tabular}

Values are given as means $\pm \mathrm{SD}$, percentages, or median (interquartile range).

Group I, 25-28 wk of gestation; Group II, 29-32 wk of gestation; Group III, 33-36 wk of gestation; Group IV, 37-40 wk of gestation; HMD, hyaline membrane disease; IUGR, intrauterine growth retardation. 
A

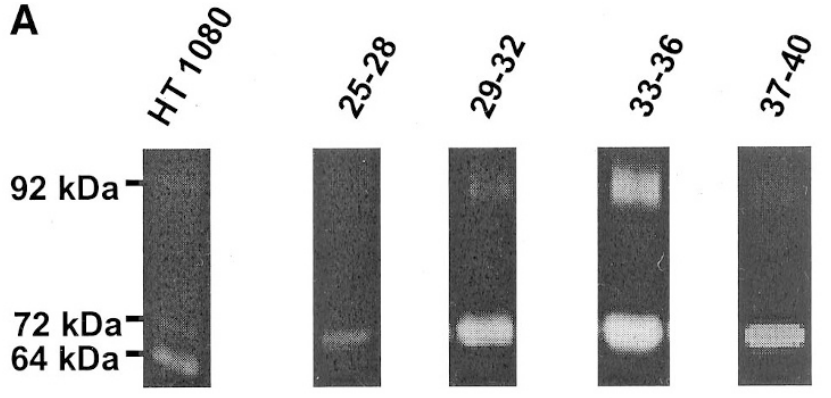

B

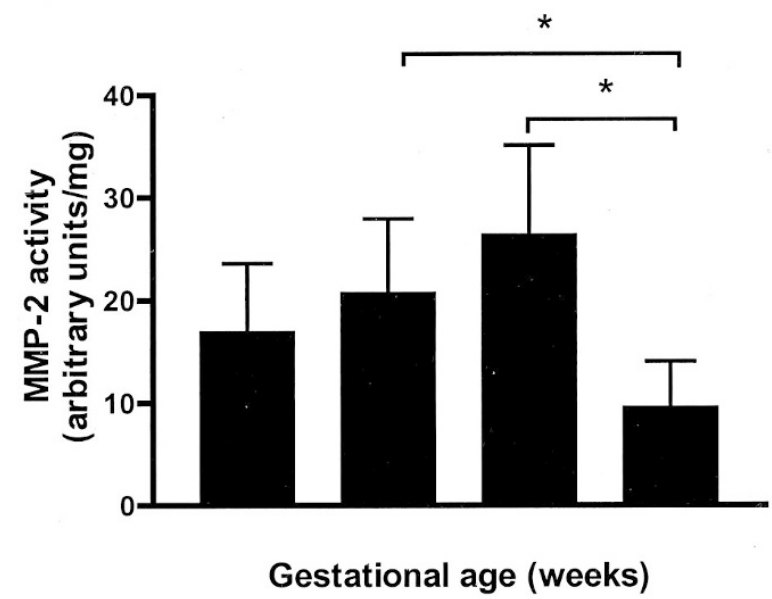

C

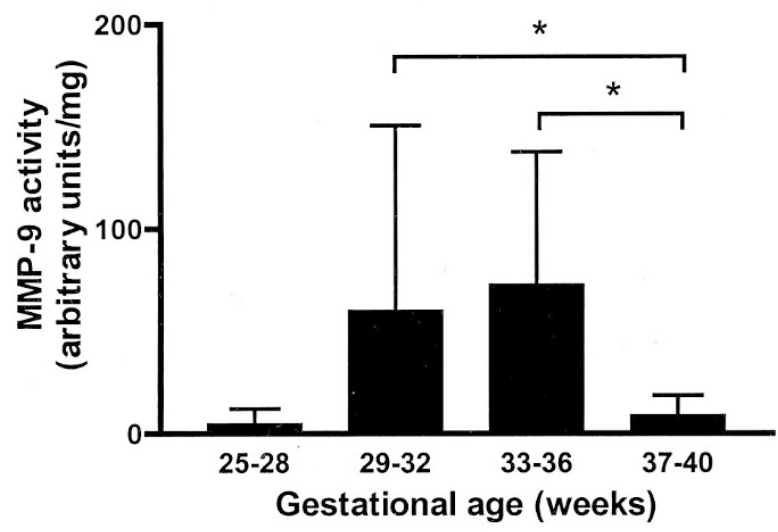

Figure 1. Developmental changes ofMMP-2 and MMP-9 plasma activity in preterm and term neonates without BPD or IVH on d 1. $(A)$ Representative zymogram cutouts. First lane HT 1080 standard showing two light bands against dark background representing MMP-2 (72 kD) and MMP-9 (92 kD). The third band is the $64 \mathrm{kD}$ active MMP-2. The following cutouts demonstrate results in patients of the four different age groups on d 1. In the 33-36 wk gestation cutting, the bands representing MMP-2 and MMP-9 activity are of higher intensity when compared with the other groups. (B) MMP-2 plasma activity peaks in group III; ${ }^{*} p<0.05$ (ANOVA). (C) MMP-9 plasma activity is significantly higher in group III; $* p<0.05$ (ANOVA).

significant postnatal changes in the plasma concentrations of TIMP-1 and TIMP-2 (Fig. 4, $A$ and $B$ ).

MMP and TIMP in neonates with BPD and/or IVH. Twelve neonates with BPD and/or IVH (group A) were matched with 12 neonates without BPD and IVH (group B) according to their gestational age ( $\leq 2 \mathrm{wk}$ limit) during the study period. There were no significant differences between the
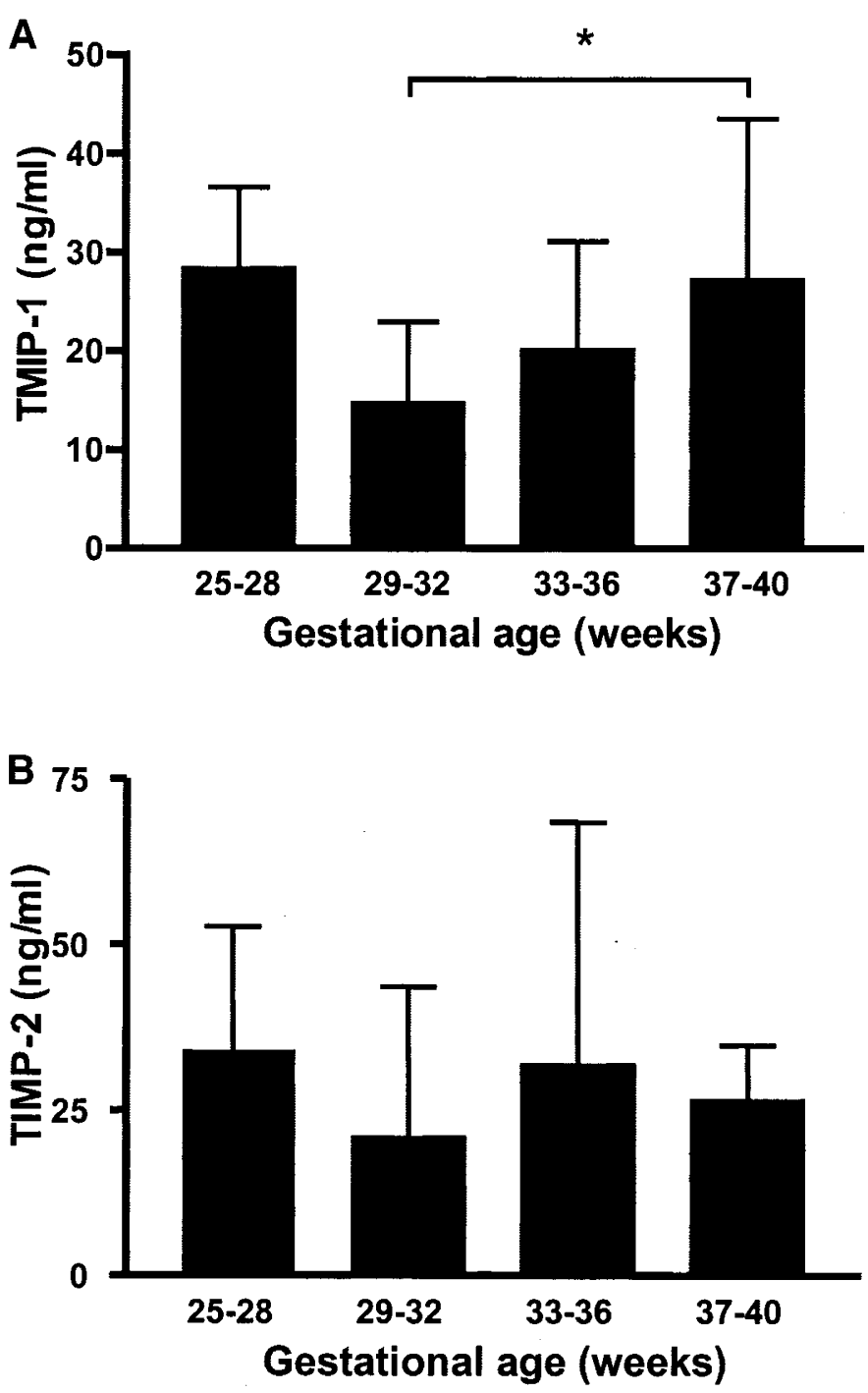

Figure 2. TIMP-1 $(A)$ and TIMP-2 $(B)$ plasma concentrations in preterm and term neonates without BPD or IVH on $\mathrm{d} 1 ;{ }^{*} p<0.05$ (ANOVA).

groups regarding the clinical characteristics other than the gestational age and duration of oxygen therapy (Table 2).

The MMP-2 plasma activity on d 1 was significantly lower in 12 neonates who were diagnosed with BPD and/or IVH when compared with the gestation-matched neonates without BPD or IVH (Fig. 5A, 5B) and the trend persisted until d 14 (d 7: $13 \pm 4$ versus $16 \pm 5$ arbitrary units/mg protein of the matched controls, $p=0.06, \beta=0.61 ; \mathrm{d} \mathrm{14}: 13 \pm 4$ versus 20 \pm 5 arbitrary units $/ \mathrm{mg}$ protein of the matched controls, $p<$ 0.05). In contrast, MMP-9 plasma activity was found to be higher in these critically ill preterm neonates on the first day of life (Fig. 5C). Determination of MMP-9 plasma activity on d 3 and $\mathrm{d} 7$ also showed significant elevations (d 3: $152 \pm 248$ versus $17 \pm 24$ and $d$ 7: $97 \pm 107$ versus $21 \pm 20$ arbitrary units/mg protein of neonates without BPD or IVH, respectively). No differences in MMP-9 plasma activities between the two groups were observed after $14 \mathrm{~d}$ of postnatal life.

The TIMP-1 plasma concentration on $\mathrm{d} 1$ in neonates with BPD and/or IVH was higher than that of gestation-matched neonates without these conditions $(p=0.05)$ (Fig. 6A), the 

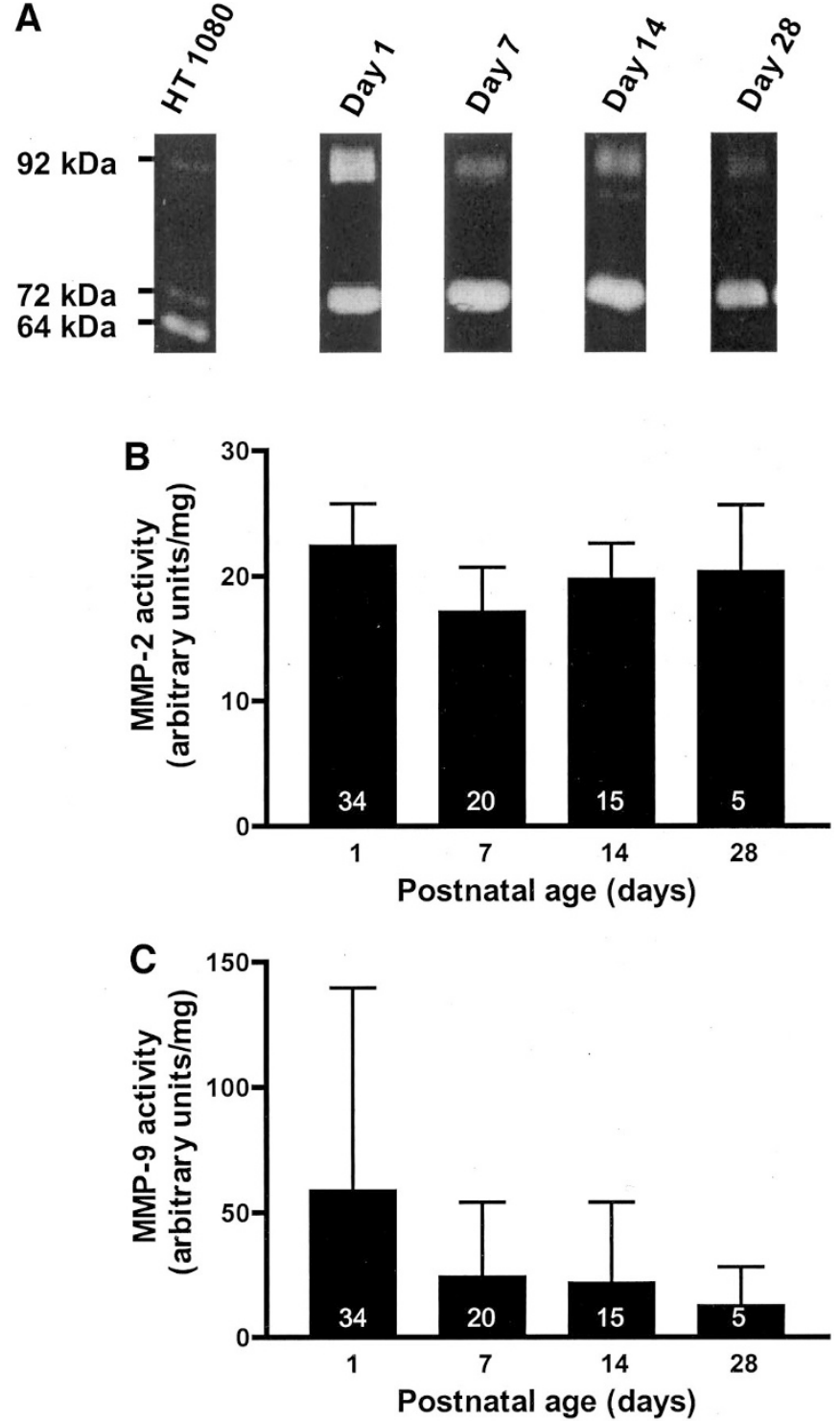

Figure 3. Postnatal changes of MMP-2 and MMP-9 plasma activities in preterm neonates (25-36 wk gestation) without BPD or IVH. Sample sizes are shown as numbers in the bars. $(A)$ Representative zymogram cutouts. First lane HT 1080 standard showing two light bands against dark background representing MMP-2 $(72 \mathrm{kD})$ and MMP-9 $(92 \mathrm{kD})$. The third band is the $64 \mathrm{kD}$ active MMP-2. Zymogram cutouts show no changes over time are seen in the $72 \mathrm{kD}$ bands, whereas the $92 \mathrm{kD}$ band on $\mathrm{d} 1$ is more intense than on any of the following days. (B) MMP-2 activity does not change during the first $28 \mathrm{~d}$ of life. (C) MMP-9 activity peaks in preterm neonates of 25-36 wk gestation on d 1, however this elevation was not significant $(p=0.08)$.

differences became not significant by d 7 and thereafter (data not shown). There were no differences observed between these two patient groups in terms of TIMP-2 plasma concentrations during the first $28 \mathrm{~d}$ of life (Fig. $6 B$ ).

The determination of MMP-2, MMP-9 plasma activities and TIMP-1 plasma concentration in neonates who developed BPD and/or IVH did not show significant postnatal changes over time. TIMP-2 plasma concentration was found to be slightly higher on $\mathrm{d} 7$ in these neonates ( $p=0.08, \beta=0.8$ ).
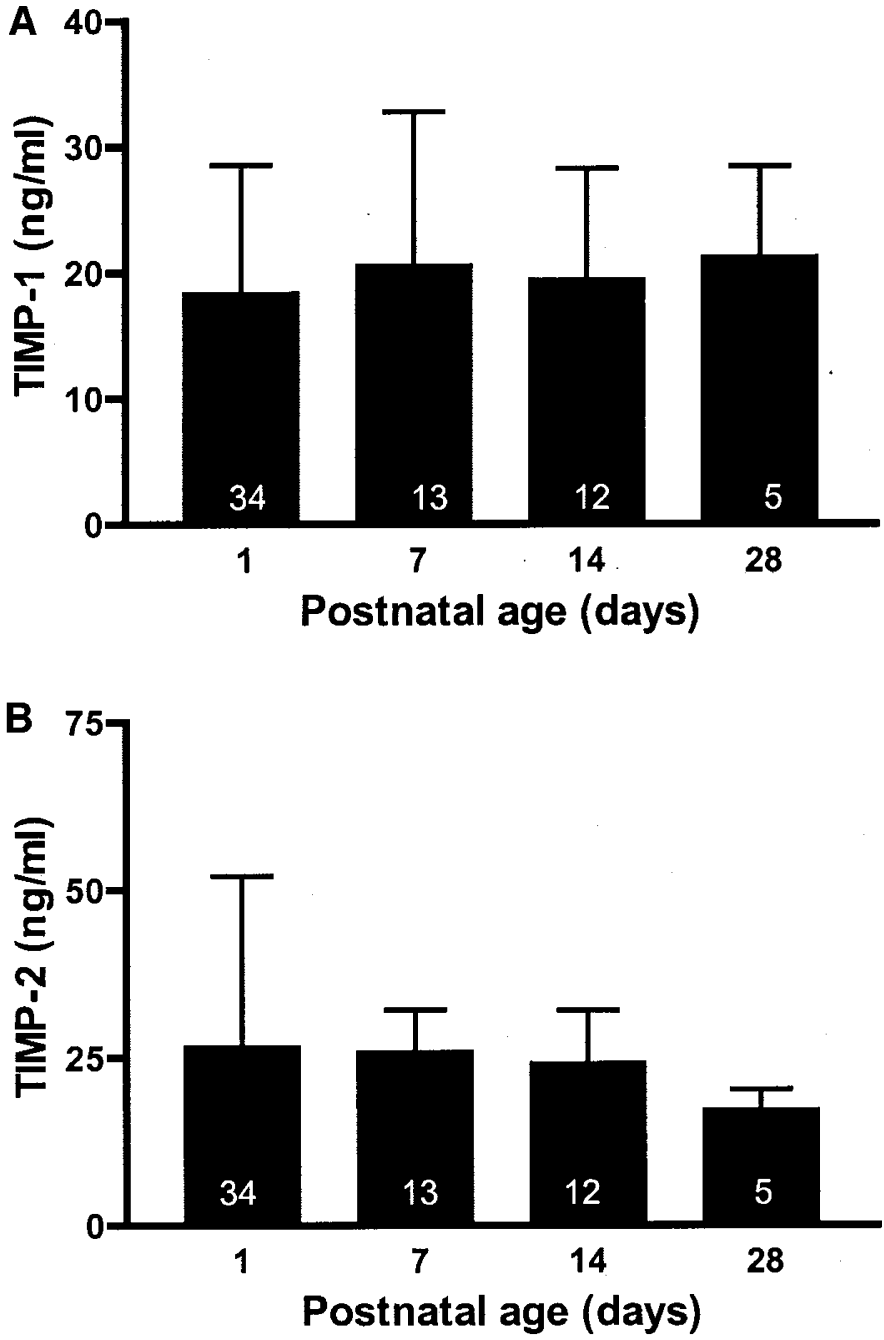

Figure 4. TIMP-1 $(A)$ and TIMP-2 $(B)$ plasma concentration in preterm neonates (25-36 wk gestation) without BPD or IVH. Sample sizes are shown as numbers in the bars.

\section{DISCUSSION}

This is the first report on normative data for MMP-2 and MMP-9 plasma activities and the plasma concentration of their inhibitors TIMP-1 and TIMP-2 in neonates of gestational age 25-40 wk. We observed 1) developmental differences between gestational age groups with the highest MMP-2 and MMP-9 plasma activities in neonates born at 33-36 wk, and TIMP-1 plasma concentration in term neonates; 2) during the first month of life, MMP-2 plasma activity and TIMP-1 and TIMP-2 plasma concentrations did not reveal changes over time, whereas MMP-9 plasma activity showed a substantial $50 \%$ decrease after postnatal age $\mathrm{d} 1$; and 3 ) compared with that of neonates without BPD or IVH, neonates who subsequently developed BPD and/or IVH had lower MMP-2 but higher MMP-9 plasma activities, and higher TIMP-1 plasma concentration during the first postnatal week.

Developmental changes of MMP-2 and MMP-9 plasma activities and TIMP-1 and TIMP-2 plasma concentrations. During feto-neonatal development, MMP play an important role in the tissue growth and morphogenesis through their 
A
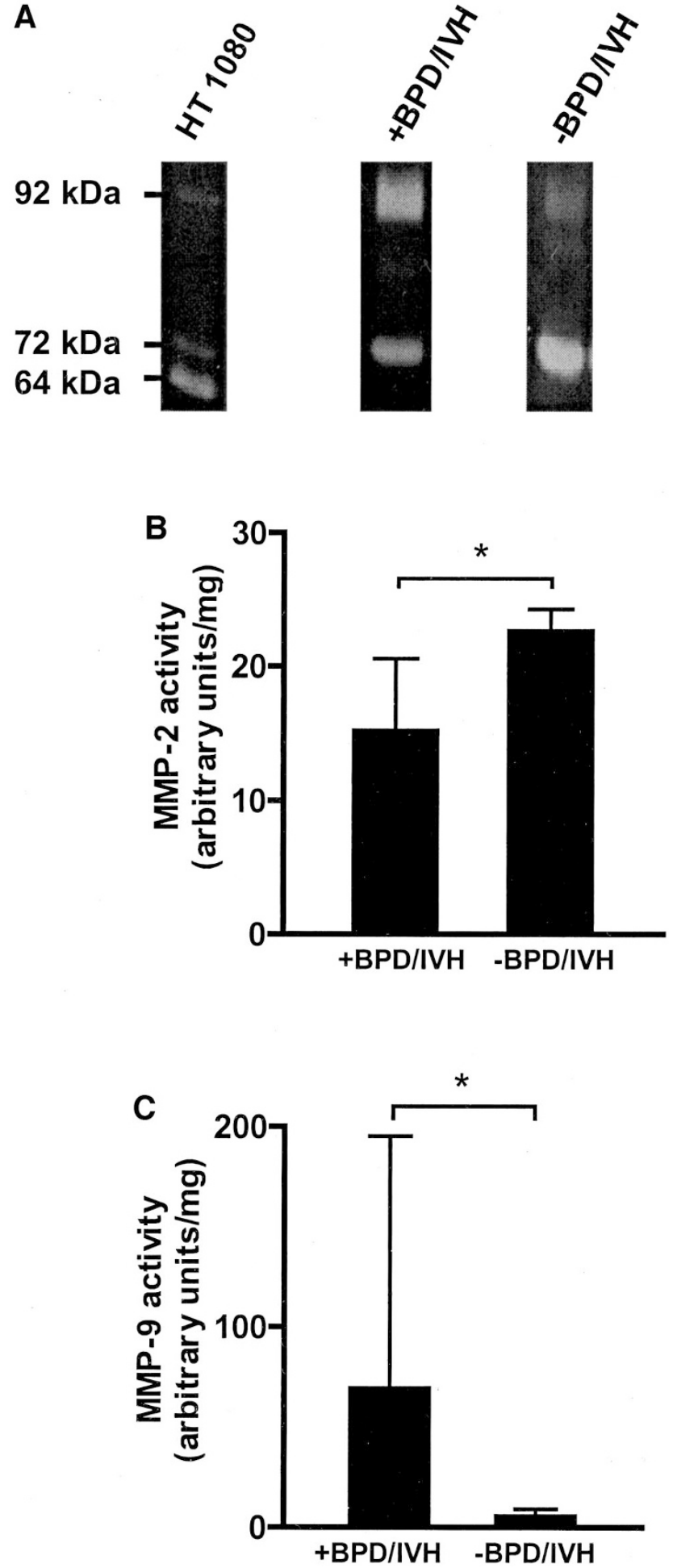

Figure 5. MMP-2 and MMP-9 plasma concentrations in preterm neonates with BPD and/or IVH and their gestational age matched controls on $\mathrm{d}$ 1. (A) Representative zymogram cutouts. First lane HT 1080 standard showing 2 light bands against dark background representing MMP-2 (72 kD) and MMP-9 (92 $\mathrm{kD})$. The third band is the $64 \mathrm{kD}$ active MMP-2. The zymogram cuttings of two patients with and without BPD or IVH demonstrating a broader band of MMP-2 in the patient without BPD or IVH and a more intense MMP-9 band in the patient with BPD and/or IVH. $(B)$ MMP-2 plasma activity in preterm neonates with BPD and/or IVH is significantly lower than those without BPD or IVH; $* p<0.05$ ( $t$ test). (C) MMP-9 plasma activity is higher in preterm neonates with BPD and/or IVH than those neonates without these conditions; $* p<0.05$ ( $t$ test $)$.
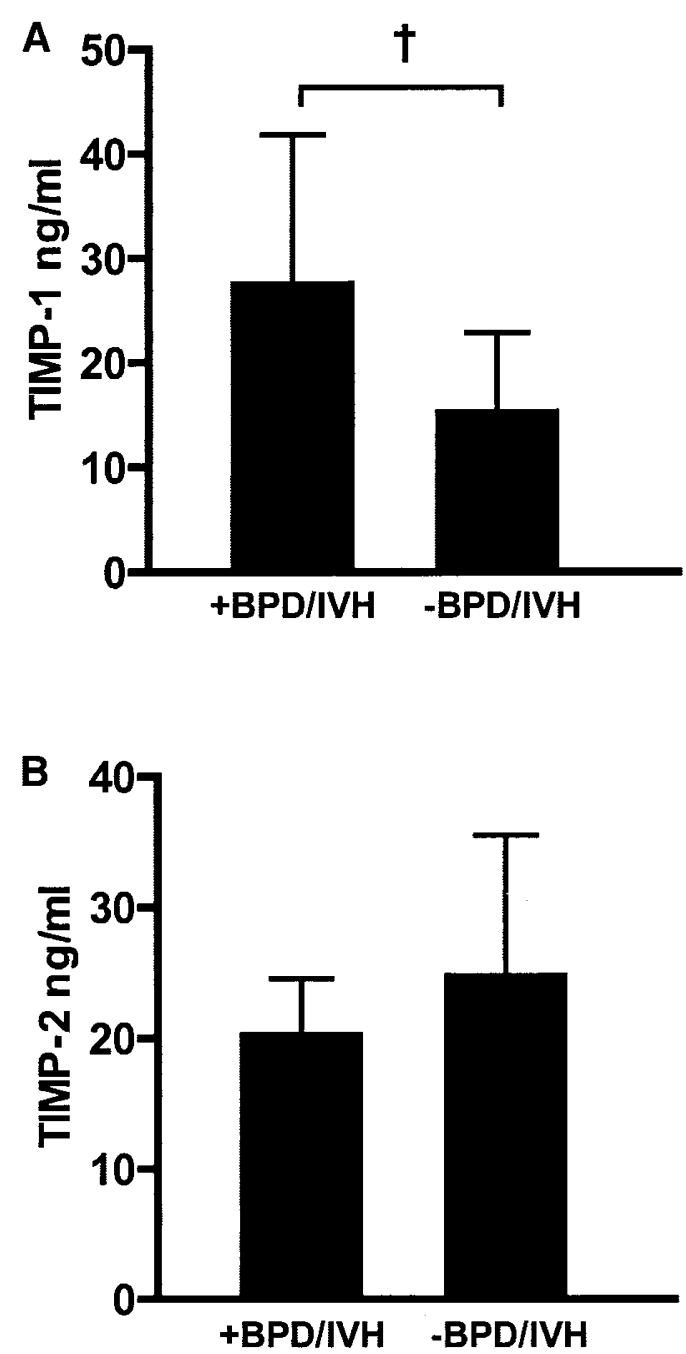

Figure 6. TIMP-1 $(A)$ and TIMP-2 $(B)$ plasma concentrations in preterm neonates with BPD and/or IVH and their gestational age matched controls on d $1 ; \dagger p<0.05$ (rank sum test).

ability to degrade the ECM (12). MMP allow cells to migrate and influence cell behavior through the alteration of the ECM. For instance, the process of angiogenesis requires endothelial cells to invade the ECM and endothelial migration has been shown to be impaired in vitro when MMP were inhibited (13). MMP also control the activation and inhibition of various mediators by proteolytic cleavage. Transforming growth factor $\beta$, which plays a role in fetal development, is cleaved in vitro by both MMP-2 and MMP-9 (14). MMP-9 has been located in neurites and seems to influence the development of the nervous system (15). High levels of MMP-9 were also found in preosteoclasts that take part in the endochondral ossification of cartilage (16).

Our findings demonstrated a gestational age-dependent increase of MMP-2 and MMP-9 plasma activity in neonates, peaking just before term pregnancy (37-40 wk). By this, near term-gestation neonates have relatively mature tissue growth and morphogenesis in the feto-neonatal development although further tissue development continues for months to years after birth. In contrast, TIMP-1 plasma concentration was found to be highest in term neonates. Although the significance is not 
clear, elevated TIMP-1 plasma concentrations may be in response to the high MMP-9 plasma activity. Despite the theoretical basis of the changes in MMP activities on the fetoneonatal development, the apparent gestational age-dependent effects may also be related to the premature pregnancy and delivery.

Postnatal changes of MMP-2 and MMP-9 plasma activities and TIMP-1 and TIMP-2 plasma concentrations. A substantial 50\% decrease of MMP-9 plasma activity was observed after $\mathrm{d} 1$ in preterm neonates of 25-36 wk gestation. The mechanism is unknown. Because MMP may be activated through oxidation (5), we speculate that postnatal adjustment from a relatively hypoxic condition in utero to higher oxygen levels ex utero may at least in part explain elevated MMP-9 plasma activity on $\mathrm{d} 1$. Preterm neonates are particularly at risk for the perinatal oxidative stress because of their lower antioxidant capacity than that of term neonates $(8,9)$. Lemke et al. (17) recently reported a surge of MMP-9 activity in rat lung tissue immediately after birth. Furthermore, MMP-9 may also be produced in inflammatory cells upon the stimulation of various mediators. Indeed, inflammatory mediators, such as IL1- $\beta$, IL-6, and tumor necrosis factor- $\alpha$, are increased during the perinatal period (18).

Interestingly, MMP-2 plasma activity as well as the concentrations of TIMP-1 and TIMP-2 showed no significant changes in their postnatal profile during the first month of life despite the ongoing process of growth and organ maturation. This is consistent with the observation by Lemke et al. (17). The postnatal growth and development of organs such as kidney, brain and lung takes months and years. Larger sample numbers and a prolonged course of investigation are therefore required for the evaluation of the role of MMP and TIMP in postnatal growth and development.

Pathologic differences of MMP-2 and MMP-9 plasma activities and TIMP-1 and TIMP-2 plasma concentrations. To our knowledge, our study was the first attempt to investigate the role of MMP and their tissue inhibitors in the pathogenesis of IVH. The confounding effects of gestational age on the patients with BPD and/or IVH (1.7 wk younger than the matched group B) are possible and may indeed contribute to the lower MMP-2 plasma activity. However, in contrast to the gestational age-dependent effects on MMP-9, patients with BPD and/or IVH had significantly higher MMP-9 plasma activities than those without BPD and/or IVH. After birth, critically ill preterm neonates are exposed to oxidative stress and their antioxidant capacity is diminished. This may cause tissue injury and lead to "oxygen radical disease of neonatology," which includes BPD, IVH, retinopathy of prematurity, and necrotizing enterocolitis $(8,9,19)$. The progression of BPD is characterized by extensive tissue remodeling of the ECM as well as the disruption of the basement membrane, resulting in increased vascular permeability and edema in the early stage. Although the local MMP-2 and MMP-9 activities were variable as sampled from the bronchoalveolar fluid (20), the respective plasma concentrations were not examined. Intracranial hemorrhage is a common complication in preterm infants (11) and involves both vascular and platelet lesions as well as hypoxia-reoxygenation injury (19). Because MMP-2 and MMP-9 are involved in the stabilization of the ECM, the regulation of vasomotor tone, and platelet aggregation, we speculate, based on our current findings, that they are related to the development of IVH. Indeed, Power et al. (7) recently reported elevated MMP-9 expression on $\mathrm{d} 1$ in a rat model of intracerebral hemorrhage. Inhibition of MMP is achieved through the formation of complexes with the active and zymogen forms of the enzymes (21). Our observations support the role of MMP and their inhibitors in the development of BPD and IVH in critically ill preterm infants. Indeed, lower MMP9/TIMP-1 ratio has been shown to be positively correlated with the progression from acute respiratory distress syndrome to lung fibrosis (22). Interestingly, the elevated MMP-9 plasma activity and TIMP-1 plasma concentration during the first week of life preceded the conventional diagnosis of BPD at $28 \mathrm{~d}$ of life.

Limitations. The small number of patients in this pilot study limited the design including the stratification of gestational age groups and a small number of relatively healthy patients in the group of 25-28 wk gestation. This factor also precludes us from performing more detailed analysis of the results. Due to ethical and practical reasons on blood sampling, we were unable to obtain consecutive samples for all enrolled patients at all time points of postnatal age. Therefore, incomplete results on MMP and TIMP at different postnatal ages could be confounded by the gestational ages of the patients at different time periods. Also, a more vigorous matching of patients could not be achieved for a proper investigation of the role of MMP plasma activities and TIMP plasma concentrations in the development of BPD and/or IVH. Nonetheless, we believe the observations in this pilot study are important and original and deserve further detailed studies on the changes of plasma MMP and their inhibitors as well as their respective roles in fetoneonatal development in a large population.

MMP-2 and MMP-9 activities were measured under in vitro conditions and these results may not represent their actual activities in vivo. In addition, we determined total levels of MMP-2 and MMP-9 plasma activity, which includes the active enzyme, the pro-enzyme, and also MMP that are bound to TIMP. Therefore, our results do not allow us to make any conclusions about the net activity of the enzymes in vivo. Similarly, the interpretation of the TIMP-1 and TIMP-2 results requires caution as the ELISA technique measures both free TIMP and TIMP bound to MMP. We also have to take into account that our measurements were done in plasma and may not directly reflect enzyme activity at tissue level.

Acknowledgments. The authors thank Walter Sumoski and the nurses of the neonatal intensive care unit and the Neonatal Research Laboratory, Royal Alexandra Hospital, for patient enrollment, collection, and timely processing of samples.

\section{REFERENCES}

1. Woessner Jr JF 1991 Matrix metalloproteinases and their inhibitors in connective tissue remodeling. FASEB J 5:2145-2154

2. Sawicki G, Sanders EJ, Murat J, Miszta-Lane H, Radomski MW 1997 Release of gelatinase A during platelet activation mediates aggregation. Nature 386:616-619 
3. Fernandez-Patron C, Radomski MW, Davidge ST 1999 Vascular matrix metalloproteinase-2 cleaves big endothelin-1 yielding a novel vasoconstrictor. Circ Res 85:906911

4. Cheung PY, Sawicki G, Wozniak M, Wang W, Radomski MW, Schulz R 2000 Matrix metalloproteinase-2 contributes to ischemia-reperfusion injury in the heart. Circulation 101:1833-1839

5. Okamoto T, Akaike T, Nagano T, Miyajima S, Suga M, Ando M, Ichimori K, Maeda H 1997 Activation of human neutrophil procollagenase by nitrogen dioxide and peroxynitrite: a novel mechanism for procollagenase activation involving nitric oxide. Arch Biochem Biophys 342:261-274

6. Murphy G, Willenbrock F 1995 Tissue inhibitors of matrix metalloendopeptidases. Methods Enzymol 248:496-510

7. Power C, Henry S, Del Bigio MR, Larsen PH, Corbett D, Imai Y, Yong VW, Peeling J 2003 Intracerebral hemorrhage induces macrophage activation and matrix metalloproteinases. Ann Neurol 53:731-742

8. Saugstad OD 1988 Hypoxanthine as an indicator of hypoxia: its role in health and disease through free radical production. Pediatr Res 23:143-150

9. Rogers S, Witz G, Anwar M, Hiatt M, Hegyi T 2000 Antioxidant capacity and oxygen radical diseases in the preterm newborn. Arch Pediatr Adolesc Med 154:544-548

10. Northway Jr WH, Rosan RC, Porter DY 1967 Pulmonary disease following respirator therapy of hyaline-membrane disease. Bronchopulmonary dysplasia. N Engl J Med 276:357-368

11. Papile LA, Burstein J, Burstein R, Koffler H 1978 Incidence and evolution of subependymal and intraventricular hemorrhage: a study of infants with birth weights less than 1,500 gm. J Pediatr 92:529-534

12. Vu TH, Werb Z 2000 Matrix metalloproteinases: effectors of development and normal physiology. Genes Dev 14:2123-2133

13. Fisher C, Gilbertson-Beadling S, Powers EA, Petzold G, Poorman R, Mitchell MA 1994 Interstitial collagenase is required for angiogenesis in vitro. Dev Biol 162:499-510
14. Yu Q, Stamenkovic I 2000 Cell surface-localized matrix metalloproteinase-9 proteolytically activates TGF-beta and promotes tumor invasion and angiogenesis. Genes Dev 14:163-176

15. Chambaut-Guerin AM, Herigault S, Rouet-Benzineb P, Rouher C, Lafuma C 2000 Induction of matrix metalloproteinase MMP-9 (92-kDa gelatinase) by retinoic acid in human neuroblastoma SKNBE cells: relevance to neuronal differentiation. J Neurochem 74:508-517

16. Blavier L, Delaisse JM 1995 Matrix metalloproteinases are obligatory for the migration of preosteoclasts to the developing marrow cavity of primitive long bones. J Cell Sci 108:3649-3659

17. Lemke RP, Zhang W, Balcerazak D, Kobayashi K, Schwingshackl A, Cheung PY, Dixon WT, Baracos VE, Greer JJ 2003 Expression and activity of MMP-2 and -9 and their inhibitors in rat lungs during the perinatal period and in diaphragmatic hernia. Exp Lung Res 29:261-276

18. Sarandakou A, Giannaki G, Malamitsi-Puchner A, Rizos D, Hourdaki E, Protonotariou E, Phocas I 1998 Inflammatory cytokines in newborn infants. Mediators Inflamm 7:309-312

19. Weinberger B, Laskin DL, Heck DE, Laskin JD 2002 Oxygen toxicity in premature infants. Toxicol Appl Pharmacol 181:60-67

20. Ricou B, Nicod L, Lacraz S, Welgus HG, Suter PM, Dayer JM 1996 Matrix metalloproteinases and TIMP in acute respiratory distress syndrome. Am J Respir Crit Care Med 154:346-352

21. Stetler-Stevenson WG, Krutzsch HC, Liotta LA 1989 Tissue inhibitor of metalloproteinase (TIMP-2). A new member of the metalloproteinase inhibitor family. J Biol Chem 264:17374-17378

22. Sweet DG, Pizotti J, Wilbourn M 1999 Matrix metalloproteinase-9 (MMP-9) in the airways of infants at risk of developing chronic lung disease. Eur Respir J 14:A248 